\title{
Reduced antibiotic prescribing for acute respiratory infections in adults and children
}

\author{
Sharon B Meropol, Zhen Chen and Joshua P Metlay
}

\begin{abstract}
Background

Recent public health efforts, including in the UK and US, have targeted decreasing unnecessary antibiotic use. In the US, prescribing for acute non-specific respiratory infections (ARIs) has decreased, but broadspectrum antibacterial prescribing has soared.
\end{abstract}

Aim

To assess recent trends in antibacterial prescribing for ARIs in the UK.

Design of study

Retrospective cohort.

Setting

The Health Improvement Network database.

Method

Outpatient ARI visits from 1 January 1990 to 31

December 2004 were selected. Outcomes were antibacterial and broad-spectrum antibacterial prescriptions per thousand person-years, and the probability of receiving an antibacterial and broadspectrum prescription conditional on an ARI visit.

\section{Results}

From 1990 to 2004, antibacterial prescribing rates for ARIs decreased from 55.0 to 30.3 prescriptions/1000 person-years for adults and from 124.8 to 46.3 prescriptions $/ 1000$ person-years for children $(P=$ $0.001)$. The probability of receiving an antibacterial prescription after an ARI visit decreased from $70.8 \%$ to $59.5 \%$ for adults and from $46.1 \%$ to $30.8 \%$ for children $(P=0.003$ and 0.007 , respectively). Antibacterial prescribing declined faster for younger than for older adults. Broad-spectrum antibacterial prescribing rates decreased from 3.8 to 2.9 prescriptions/1000 personyears for adults and from 5.2 to 2.2 prescriptions $/ 1000$ person years for children $(P=0.005$ and 0.003 , respectively). The probability of broad-spectrum prescribing did not demonstrate a significant linear trend for adults $(P=0.16)$, and decreased for children $(P=0.01)$.

\section{Conclusion}

UK antibacterial prescribing for ARIs has declined, similar to US trends, but there was no concomitan increase in low broad-spectrum prescribing. The success of UK strategies for limiting antimicrobial use has implications for programmes in other countries.

\section{Keywords}

anti-infective agents; databases; health services research; prescriptions; respiratory tract infections.

\section{INTRODUCTION}

Resistance to antibacterial medications among community-acquired pathogens is a growing public health threat. ${ }^{1-5}$ Key drivers are the volume and type of antibacterials used in ambulatory settings..$^{6-8}$ Antibacterials are often prescribed for acute nonspecific respiratory infections (ARIs), which they are unlikely to benefit. ${ }^{9,10}$ Reducing such use can slow, or even reverse resistance rates. ${ }^{11,12}$ US and UK campaigns have discouraged unnecessary antibacterial use.,.$^{2,13-16}$ Recent US data have demonstrated decreased unnecessary adult and child use, but US broad-spectrum antibacterial use for adult and child ARIs more than doubled during the 1990s and continues to increase..$^{17-23}$ UK studies have similarly shown decreased ARI diagnoses and overall antibacterial use for all ages, ${ }^{24-28}$ but have

SB Meropol, MD, MSCE, research fellow in clinical epidemiology, Center for Clinical Epidemiology and Biostatistics, Penn Center for Education and Research on Therapeutics, and Department of Biostatistics and Epidemiology; Z Chen, PhD, assistant professor of biostatistics, Center for Clinical Epidemiology and Biostatistics and Department of Biostatistics and Epidemiology, University of Pennsylvania School of Medicine, Philadelphia, PA, US. JP Metlay, MD, PhD, associate professor of medicine and epidemiology, Center for Clinical Epidemiology and Biostatistics, Penn Center for Education and Research on Therapeutics, Department of Biostatistics and Epidemiology, and Department of Medicine, University of Pennsylvania School of Medicine and research associate and staff physician, VA Medical Center, Philadelphia, PA, US.

Address for correspondence

Sharon B Meropol, 108 Blockley Hall, 423 Guardian Drive, Center for Clinical Epidemiology and Biostatistics, University of Pennsylvania School of Medicine, Philadelphia, PA 19104, US.

E-mail: meropols@mail.med.upenn.edu

Submitted: 4 September 2008; Editor's response: 11 February 2009; final acceptance: 18 March 2009.

(C) British Journal of General Practice

This is the full-length article of an abridged version published in print. Cite this article as: Br J Gen Pract 2009; DOI: 10.3399/bjgp09X472610. 
provided limited information regarding trends in adult and child antibacterial and broad-spectrum antibacterial use for ARIs.

The objective of this study was to assess recent UK trends in overall and broad-spectrum antibacterial drug use for adult and child ARIs. It was hypothesised that overall use declined, that this decline varied by age, and, unlike recent US patterns, that there was no concomitant increase in broad-spectrum drug utilisation.

\section{METHOD}

\section{Study design}

This retrospective cohort study utilised de-identified data from a large UK primary care electronic medical record database, The Health Improvement Network (THIN). ${ }^{29}$ Within the UK, approximately $98 \%$ of the population is registered with a GP who is responsible for almost the entirety of the patient's medical care. Data collection commenced in 1985 through the General Practice Research Database (GPRD), originally established by the EPIC Research Company for research purposes; THIN, introduced by EPIC in 2002, includes longitudinal data from many original GPRD practices and continues to enrol additional practices with ongoing data collection from general practices throughout the UK. ${ }^{29,30}$

THIN includes demographics, physician and nurse visits, diagnoses, and prescriptions. Prescriptions are generated by data entry, and GP practices are responsible for most prescribing, with virtually $100 \%$ capture of prescription data. Practitioners are trained in data entry, and their data quality is reviewed on an ongoing basis for quality and completeness. ${ }^{29}$ Studies have confirmed good validity regarding documented diagnoses, ${ }^{31,32}$ prescriptions, ${ }^{32}$ and specialists' information. ${ }^{33}$

\section{Study population}

The population of interest included permanently registered members of computerised THIN practices, utilising THIN data as of September 2007, describing 4.85 million patients from 326 practices, including $>32$ million person-years. The study used valid available data from 1 January 1990, or the date of practice computerisation if later, to 31 December 2004, or the latest date of data collection for that practice.

THIN records birth year for all patients and birth month for children $<15$ years of age. In this study, adults were defined as individuals $\geq 18$ years of age on the day of the ARI visit and children as being $<18$ years of age, according to THIN recorded birth dates.

A cohort of ARI visits from 1 January 1990 to 31 December 2004 was selected using Read diagnostic codes for acute non-specific respiratory infections, chosen to represent conditions that are typically viral

\section{How this fits in}

Previous studies using data from other developed countries have shown a

recent decrease in unnecessary use of antibacterial drugs for acute non-specific respiratory infections (ARIs) in adults and children, but a concomitant large increase in broad-spectrum antibacterial use. While studies using UK data have shown decreased antibacterial use in general, the relationship between adult and child ARls and antibacterial and broad-spectrum antibacterial drug use in the UK has not yet been described. This study suggests that overall antibacterial use for ARIs in the UK has recently decreased, with no concomitant increase in already quite low broad-spectrum antibacterial drug use. Understanding the reasons underlying this very different drug use pattern between the US and the UK could help the development of strategies to further improve antibacterial drug use in many countries that are currently

implementing programmes to reduce unnecessary antibiotic use.

in origin and unlikely to respond to antibacterials (Appendix 1). Conditions for which some guidelines recommend antibacterials, such as otitis media and sinusitis, were excluded. Because data from multiple visits within the same ARI episode may be highly correlated, adjacent visits were grouped within a 2week window for the primary analysis; sensitivity analysis explored the impact of considering adjacent visits independently. The study also explored the impact of not including the visits with bronchitis diagnoses. As results of these sensitivity analyses were identical to the primary analysis, only primary analysis results are presented.

\section{Outcome classification}

The outcome of interest was receiving any antibacterial medication prescription within 1 day of an ARI visit. Drugs of interest included oral antibacterials typically used for respiratory infections. Topical, vaginal, ophthalmologic, otic, and parenteral antibacterials were excluded, as well as those typically used for tuberculosis, fungal, and parasitic infections. Amoxicillin/clavulanate, azithromycin, clarithromycin, fosfomycin, secondand third-generation cephalosporins, and quinolones were classified as broad spectrum, and all others, including penicillin, ampicillin, amoxicillin, tetracycline, doxycycline, erythromycin, and trimethoprim/sulfamethoxazole, as narrow-spectrum medications. ${ }^{23,34}$

\section{Exposure classification}

The main exposure was visit year, considered individually for all analyses.

\section{Covariates}

For children, age was stratified as $0-<5$ and $\geq 5$ years of age. For adults, age was stratified as $18-<65$ (younger age group) and $\geq 65$ years (older age group). These age categories are clinically relevant (that is, 


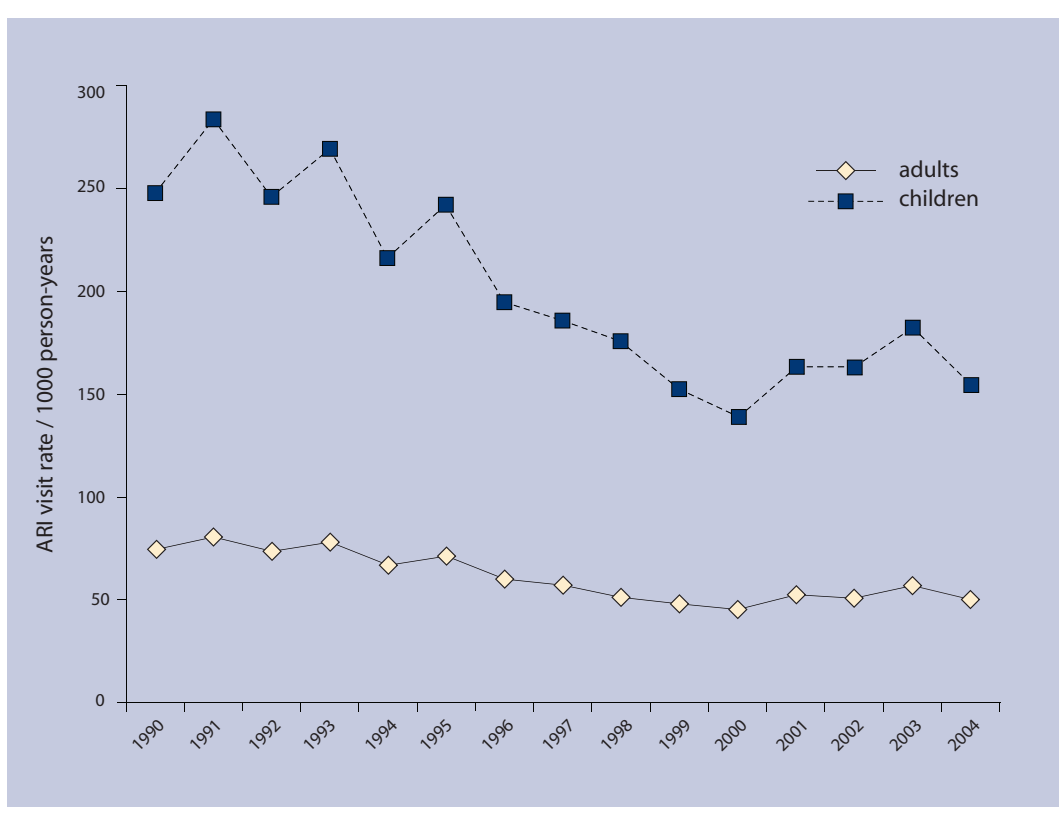

Figure 1. Adult and child visit rates per 1000 person-years.

pre-school versus school-aged children), are in line with those used by the US National Center for Health Statistics, ${ }^{35}$ and can facilitate comparisons with US Medicare data for adults aged $\geq 65$ years. Other covariates included sex, the number of comorbidities by the day of the ARI visit, the number of different classes of prescribed medications, and the number of visits within the year before the ARI visit. ${ }^{36-38}$ As changes in drug use could vary by age; the study also tested for an interaction between year and age category.

\section{Analysis}

Figure 2. Adult and child antibacterial prescribing Trends in ARI visit rates and antimicrobial prescribing rates over the study period were described using rates per 1000 person-years. Cuzick's non-parametric test for trend across

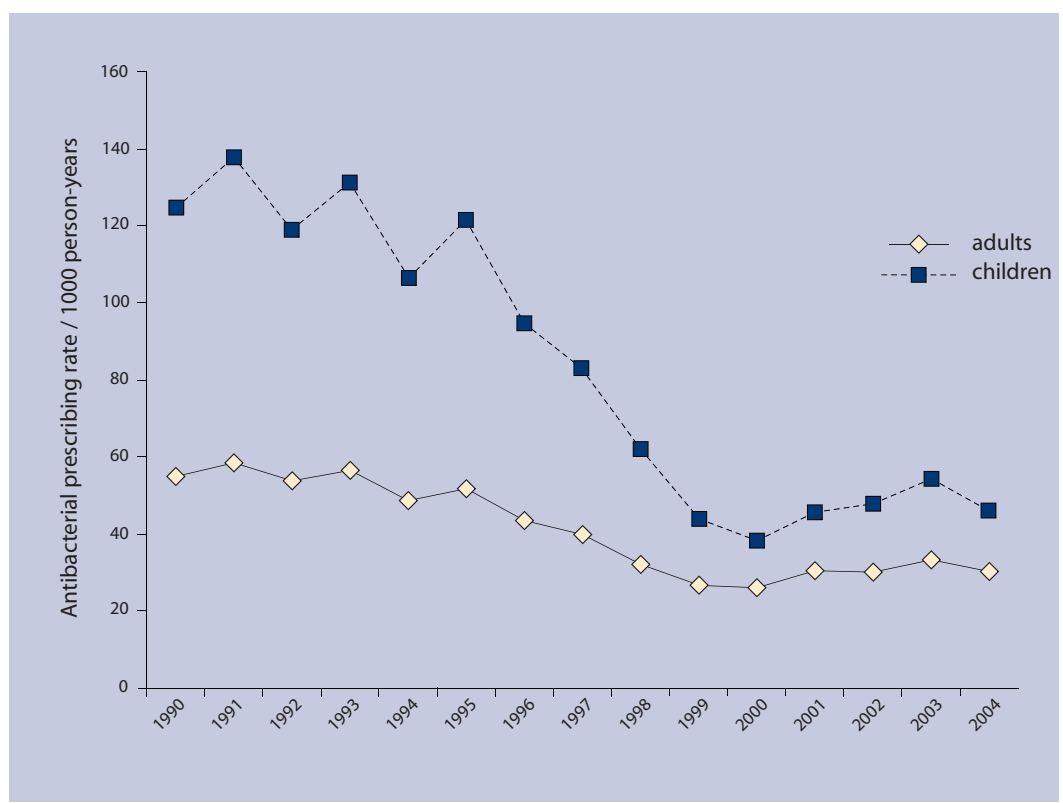

ordered groups by individual visit year. Person time was calculated using each patient's THIN birth date, practice enrolment date, date of transfer out of practice, and/or date of death, and each practice's computerisation and/or last data-collection date, as appropriate.

Generalised linear models were used to model the probability of an antibacterial prescription, conditional on an ARI visit. To predict probabilities, a Poisson distribution and logarithm link function were used in the generalised linear models, with robust variance estimates. $^{39}$ Separate adult and pediatric models were adjusted for clustering by patient and practice using generalised estimating equations. ${ }^{40,41}$ The probability that an antibacterial was prescribed was modelled, first using models adjusted only for year. Probabilities of antibacterial use for each year are reported, as well as the trend across year investigated using Cuzick's test. The probability of antibacterial prescribing was modelled using fully adjusted models, with year as a linear term, including all covariates described above, to report the adjusted trend across year, described as an incidence rate ratio (IRR) for each successive year. The study also explored the age-year interaction using the fully adjusted model including the interaction between age category and categorical year, and tested whether interaction terms were significant, using the deviance difference test. ${ }^{42}$ If interaction terms were statistically significant, IRRs are reported for each successive year stratified by age category.

A parallel set of analyses was performed for broadspectrum antibacterial drugs, including trends in prescribing rates and the probability of antibiotic prescriptions conditional on ARI visits. Analyses were performed using Stata (version 9, StataCorp LP).

\section{RESULTS}

\section{$A R I$ visit rate}

A total of $1342365 \mathrm{ARI}$ visits in 745044 adults followed for 22741927 person-years and 1117596 ARI visits in 453584 children followed for 5831438 person-years were identified. For adults, the ARI visit rate during 1990 was 74.5 visits per 1000 personyears and by 2004 it was 50.2 visits per 1000 personyears (Figure 1). For children, the ARI visit rate during 1990 was 247.9 per 1000 person-years and by 2004 it was 154.5 per 1000 person-years (Figure 1). Visit rates decreased over the study period for adults and children $(P=0.001)$.

\section{ARI antibacterial prescription rate}

For adults, in 1990, the antibacterial prescription rate for ARI visits was 55.0 per 1000 person-years, and by 2004 it was 30.3 per 1000 person-years $(P=$ 0.001) (Figure 2). For children, in 1990 the 
antibacterial prescription rate was 124.8 per 1000 person-years, and by 2004 it was 46.3 per 1000 person-years $(P=0.001)$.

\section{Probability of antibacterial drug prescribing conditional on ARI visit}

For adults, during $199071 \%$ of ARI visits were associated with an antibacterial prescription, and by 2004 , 59\% of visits were associated with antibacterials $(P=0.003)$ (Figure 3).

Using the fully adjusted model for adults, adjusting for sex, age category, year, comorbidities, number of medications, and number of visits, there was a significant decrease in the probability of antibacterial prescribing for each successive year, with an IRR of 0.980 (95\% confidence interval $[\mathrm{Cl}]=0.979$ to 0.980 , $P<0.001)$.

Using the fully adjusted model for adults, and including the year-age category interaction, older adults were initially less likely to receive antibacterials $(P<0.001$ comparing older and younger adults in every year), until 1998 when older and younger adults were equally likely to receive antibacterials $(P=0.930$ older versus younger adults); after 1998 , older adults were more likely to receive antibacterials $(P<0.001$ comparing older and younger adults in every year after 1998). Both age categories experienced significant declines in the probability of antibacterial prescribing over the study period; the IRR for year for ages $18-<65$ years was $0.977(95 \% \mathrm{Cl}=0.977$ to 0.978$)$ and for $\geq 65$ years was $0.988(95 \% \mathrm{Cl}=0.987$ to 0.988$)$. The age-year interaction was significant $(P<0.001)$, indicating that, over time, antibacterial prescribing declined more steeply for younger than for older adults.

For children, during $1990,46 \%$ of ARI visits were associated with antibacterial prescriptions, and by $2004,31 \%$ of $\mathrm{ARI}$ visits were associated with antibacterials $(P=0.007)$ (Figure 3$)$.

Using the fully adjusted model for children, there was a significant decrease in the probability of antibacterial prescribing for each successive year, with an IRR of $0.960(95 \% \mathrm{Cl}=0.959$ to 0.960 , $P<0.001)$.

Using the fully adjusted model for children, and including the year-age category interaction, older children were $30-40 \%$ more likely than younger children to receive antibacterial prescriptions in every study year $(P<0.001$ comparing older versus younger children in each study year). Both age categories experienced significant declines in antibacterial drug prescribing over the study period; the IRR for year for ages $0-<5$ years was 0.959 (95\% $\mathrm{Cl}=0.958$ to 0.959$)$ and for $\geq 5$ years was 0.962 (95\% Cl $=0.961$ to 0.963 ), indicating that the rate of decline was similar in both age groups. While the

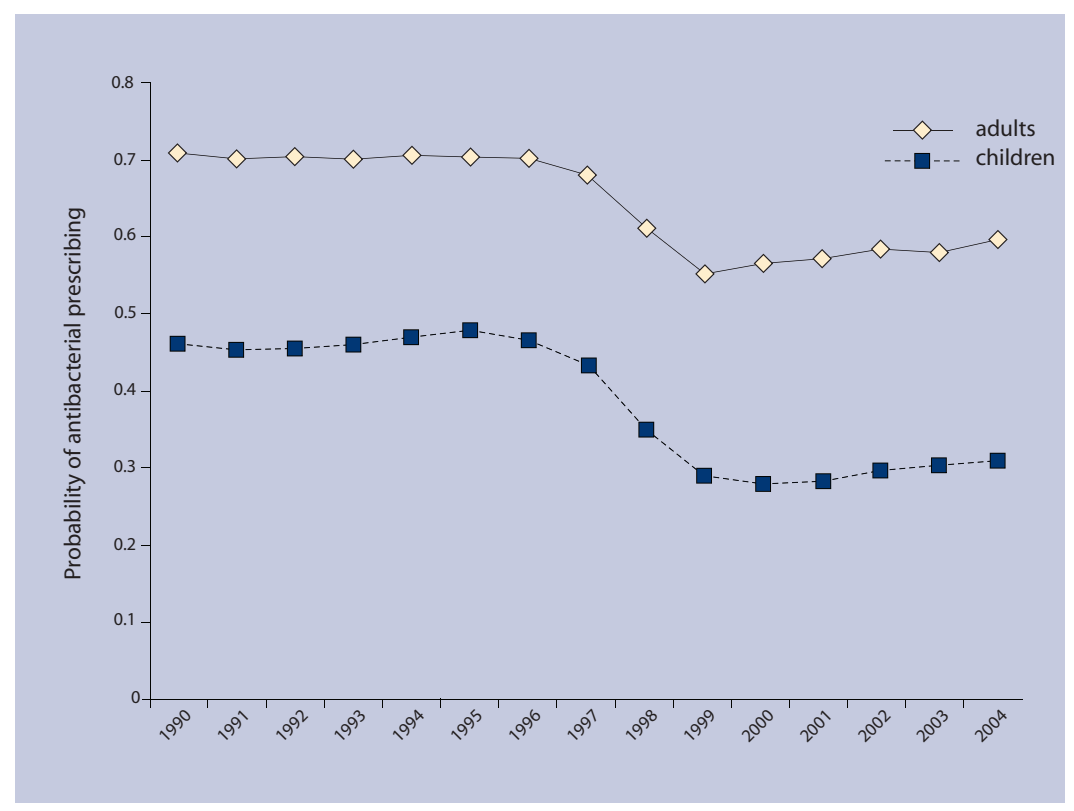

age-year interaction term was statistically significant, the effect of year, and thus its public health relevance, was essentially the same in both age groups.

\section{ARI broad-spectrum antibacterial prescribing rate}

For adults, the broad-spectrum antibacterial drug prescription rate during 1990 was 3.8 prescriptions per 1000 person-years, and by 2004 was 2.9 prescriptions per 1000 person-years $(P=0.005)$ (Figure 4). For children, the broad-spectrum antibacterial prescription rate during 1990 was 5.2 prescriptions per 1000 person-years, and by 2004 was 2.2 prescriptions per 1000 person-years $(P=$ 0.003) (Figure 4).

Figure 3. Probability of antibacterial drug prescribing after ARI visit.

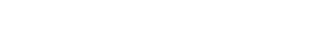

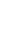

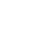




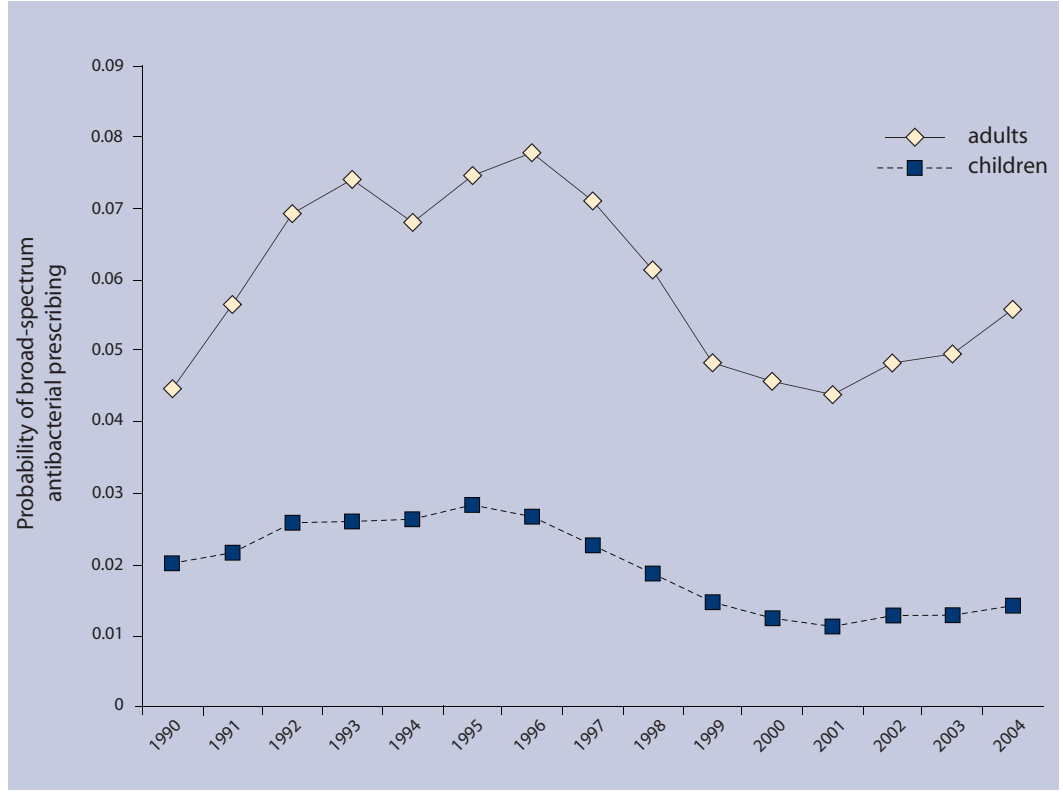

Figure 5. Probability of broad-spectrum antimicrobial drug prescribing after ARI visit.

Probability of broad-spectrum antibacterial prescribing conditional on ARI visit

For adults, during $1990,4.4 \%$ of ARI visits were associated with broad-spectrum antibacterials; this portion peaked at $7.8 \%$ by 1996 and then decreased to $5.6 \%$ by $2004(P=0.16$ for linear trend over study period) (Figure 5).

Using the fully adjusted model for adults, there was a small decline in the probability of broadspectrum antibacterial prescribing for each successive year, with an IRR of $0.960(95 \% \mathrm{Cl}=$ 0.960 to $0.970, P<0.001)$.

For children, during $1990,2.0 \%$ of visits were associated with broad-spectrum antibacterial prescriptions (Figure 5); this percentage peaked at $2.8 \%$ in 1995 and then decreased to $1.4 \%$ by $2004(P$ $=0.01$ ).

Using the fully adjusted model for children, there was a small decline in the probability of broadspectrum antibacterial prescribing for each successive year, with an IRR of $0.950(95 \% \mathrm{Cl}=0.940$ to $0.950, P<0.001$ ).

\section{DISCUSSION}

\section{Summary of main findings}

This study demonstrated that antibacterial drug prescribing for ARIs decreased in the UK for adults and children from 1990 to 2004. The decline in antibacterial use was faster for both older and younger children than for adults, although use in younger adults declined faster than for older adults. Possible reasons for these differences include: the influence of the pneumococcal conjugate vaccine on the perceived risk of child bacterial illness ${ }^{43-45}$ and on parents' health through herd effects; ${ }^{45,46}$ a potentially initially wider pool of unneeded antibacterial use in younger individuals; and a possible differential effect of public educational efforts regarding antibacterial use for young adults, influencing their own use and that of their children. The relative contributions of each of these or other factors to the study results are unknown.

Despite decreasing antibacterial use for ARIs, a concomitant increase in broad-spectrum antibacterial prescribing was not observed. In fact, encouraging evidence was found for low and recently decreasing broad-spectrum antibacterial use for UK adults and children.

\section{Strengths and limitations of the study}

Strengths of this study relate to the use of THIN data. Advantages of THIN versus claims data are THIN's direct links to longitudinal clinical data and that THIN does not depend on billing or insurance status. Advantages of THIN versus survey data are that THIN is a $100 \%$ sample of practice patients and that the medical record itself is the data-collection form.

Potential limitations of this study include that some antibacterials may have been missed, for example, telephoned prescriptions without an associated ARI visit. Secondly, concomitant trends in diagnosis and antibiotic use were not measured for other infectious diagnoses. It is possible that there has been a diagnostic shift, and that caregivers are now diagnosing more infections for which antibiotics are indicated. However, other studies from the UK and the US have not found a concomitant increase in antibiotic prescribing for other infectious diagnoses, in fact, visits and antibiotic use for other infectious diagnoses have also decreased in the US and the UK. ${ }^{22,47,48}$ Thirdly, visit grouping may have misclassified some unexposed visits as exposed and falsely inflated the antibacterial use estimates; however, the sensitivity analysis considering ungrouped visits showed similar results. Next, the observational study does not make it possible to address which policies or clinical trends caused the observed changes. Finally, the study did not address outcomes of antibacterial use and could not directly assess prescriptions' appropriateness.

\section{Comparison with existing literature}

The population rates of ARI visits observed in this study are similar to those previously reported for US adults and children..$^{20,22,49}$

The trends in overall antibacterial use observed are comparable to US trends. Using National Ambulatory Medical Care Survey (NAMCS) data, Roumie et al reported that antibacterial prescribing for adult ARIs declined from $60 \%$ in $1995-1997$ to $43 \%$ in 1999-2000. ${ }^{20}$ Steinman et al also used NAMCS data to report decreased antibacterial prescribing for adult ARIs from $56 \%$ in 1991 to $43 \%$ in $1999 .{ }^{21}$ The decline in UK antibiotic prescribing for adult ARIs 
demonstrated in this study, from $71 \%$ in 1990 to $59 \%$ in 2004 , is similar to these US reports. Similarly, Steinman et al reported declining antibacterial use for child ARIs, from $41 \%$ in 1991 to $21 \%$ in $1999 .{ }^{21}$ In the present study cohort, child antibiotic use for ARIs decreased from $46 \%$ in 1990 to $31 \%$ in 2004 . Using updated NAMCS data, Grijalva et al recently reported continued decreasing overall antibacterial use and increasing broad spectrum antibacterial use for ARIs in US adults and children. ${ }^{17}$

The study finding of low and recently decreasing use of broad-spectrum antibacterials for adults and children in the UK is quite different from US trends, evidence that recent UK campaigns to enhance judicious antibiotic use may be paying off. ${ }^{14-17}$

\section{Implications for future research}

Reasons for the large discrepancies in trends in broad-spectrum antibacterial use between the UK and US are unknown, but could relate, at least in part, to differences in healthcare delivery. US health care is managed by a mix of privately and publicly financed mechanisms, emphasising a competitive business model. Prescribing is influenced by separate formularies for each of thousands of individual health plans, and pharmaceutical industry promotion to physicians and the public. The US Centers for Disease Control and Prevention's (CDC's) 'Get Smart' campaign targeted parents with the message that using antibacterials for ARls put their children at greater risk of a future resistant infection. UK health care is managed by a government-financed national system which sets explicit priorities to enhance public health through specific incentives. Medications are managed with national guidelines and with formularies and performance monitoring of antibiotic prescribing at the local level. The UK's campaign, 'Antibiotics: Don't Wear Me Out', targeted the general public with the message that controlling antibiotic resistance benefits everyone.

Successful strategies to further reduce antibiotic overuse are likely to have strong central leadership, with explicit priorities emphasising societal benefit, and to be supported by robust financial and regulatory incentives. Professional and public education, while necessary, are usually not sufficient to change behaviour; successful strategies for improving antibacterial use are usually multifaceted. More data are needed regarding outcomes of strategies to reduce antibacterial use and whether decreasing use may be affecting trends in antimicrobial resistance.

\section{Funding body}

This project was supported by grant number U18-HS016946 from the Agency for Healthcare Research and Quality and by grant number F32-Al-073015-01A1 from the National Institute for Allergy and Infectious Diseases. The content is solely the responsibility of the authors and does not necessarily represent the official views of the Agency for Healthcare Research and Quality

\section{Ethics committee}

The limited dataset used in this study is covered by a data use agreement between the Center for Clinical Epidemiology and Biostatistics at the University of Pennsylvania and EPIC Database Research Company. This study was granted exempt status by the University of Pennsylvania Institutional Review Board, and approval by both the University of Pennsylvania THIN User Committee and EPIC Database Research Company in the UK

\section{Competing interests}

The authors have stated that there are none

\section{Acknowledgements}

Zhen Chen's current affiliation: National Institute of Child Health and Human Development, Rockville. This work was presented, in part, at the Pediatric Academic Societies Annual Meeting, May 4, 2008, Honolulu, HA, and the 4th Annual International Conference on Pharmacoepidemiology and Therapeutic Risk Management, 20 August, Copenhagen, Denmark. EPIC is a licence holder of an historical part of the GPRD dataset. GPRD is owned by the Secretary of State for Health and is managed on his behalf by the GPRD Group at the MHRA. The name GPRD is a trade mark of the GPRD group.

\section{Discuss this article}

Contribute and read comments about this article on the Discussion Forum: http://www.rcgp.org.uk/bjgp-discuss

\section{REFERENCES}

1. Lonks JR, Garau J, Gomez L, et al. Failure of macrolide antibiotic treatment in patients with bacteremia due to erythromycin-resistant Streptococcus pneumoniae. Clin Infect Dis 2002; 35(5): 556-564.

2. Interagency Task Force on Antimicrobial Resistance. A public health action plan to combat antimicrobial resistance. Part 1: domestic issues. Atlanta: Interagency Task Force on Antimicrobial Resistance, 1999. http://www.cdc.gov/drugresistance/actionplan/index.htm (accessed 23 Mar 2009)

3. Austrian R. Confronting drug-resistant pneumococci. Ann Intern Med 1994; 121(10): 807-809.

4. Klugman KP. Pneumococcal resistance to antibiotics. Clin Microbiol Rev 1990; 3(2): 171-196.

5. Metlay JP, Hofmann J, Cetron MS, et al. Impact of penicillin susceptibility on medical outcomes for adult patients with bacteremic pneumococcal pneumonia. Clin Infect Dis 2000; 30(3): 520-528.

6. Lipsitch $\mathrm{M}$. The rise and fall of antimicrobial resistance. Trends Microbiol 2001; 9(9): 438-444.

7. Austin DJ, Kristinsson KG, Anderson RM. The relationship between the volume of antimicrobial consumption in human communities and the frequency of resistance. Proc Natl Acad Sci U S A 1999; 96(3): 1152-1156.

8. Lipsitch M, Samore MH. Antimicrobial use and antimicrobial resistance: a population perspective. Emerg Infect Dis 2002; 8(4): 347-354.

9. Gonzales R, Steiner JF, Sande MA. Antibiotic prescribing for adults with colds, upper respiratory tract infections, and bronchitis by ambulatory care physicians. JAMA 1997; 278(11): 901-904.

10. Nyquist AC, Gonzales R, Steiner JF, Sande MA. Antibiotic prescribing for children with colds, upper respiratory tract infections, and bronchitis. JAMA 1998; 279(11): 875-877.

11. Seppala H, Klaukka T, Vuopio-Varkila J, et al. The effect of changes in the consumption of macrolide antibiotics on erythromycin resistance in group A streptococci in Finland. Finnish Study Group for Antimicrobial Resistance. N Engl J Med 1997; 337(7): 441-446.

12. Stephenson J. Icelandic researchers are showing the way to bring down rates of antibiotic-resistant bacteria. JAMA 1996; 275(3): 175

13. US Centers for Disease Control and Prevention, US Department of Human Services. Get SMART: know when antibiotics work. http://www.cdc.gov/getsmart/ (accessed 23 Mar 2009).

14. Standing Medical Advisory Committee Sub-Group on AntiMicrobial Resistance. The path of least resistance. http://www.advisorybodies.doh.gov.uk/smacl.htm (accessed 23 Mar 2009). 
15. Department of Health. Antibiotics: don't wear me out. London: Department of Health, 1999.

www.dh.gov.uk//PublicationsAndStatistics/Publications/Publications PolicyAndGuidance/PublicationsPolicyAndGuidanceArticle/fs/en?C ONTENT_ID=4007460\&chk=VHEgjn (accessed 23 Mar 2009).

16. Ashworth M, Golding S, Majeed A. Prescribing indicators and their use by primary care groups to influence prescribing. J Clin Pharm Ther 2002; 27(3): 197-204.

17. Grijalva, CG, Nuorty, JP, Griffin, MR. Antibiotic prescription rates for acute respiratory tract infections in US ambulatory settings. JAMA 2009; 302(7): 758-766.

18. Halasa NB, Griffin MR, Zhu Y, Edwards KM. Decreased number of antibiotic prescriptions in office-based settings from 1993 to 1999 in children less than five years of age. Pediatr Infect Dis J 2002; 21(11): 1023-1028.

19. McCaig LF, Besser RE, Hughes JM. Antimicrobial drug prescription in ambulatory care settings, United States, 1992-2000. Emerg Infect Dis 2003; 9(4): 432-437.

20. Roumie CL, Halasa NB, Grijalva CG, et al. Trends in antibiotic prescribing for adults in the United States — 1995 to 2002. J Gen Intern Med 2005; 20(8): 697-702.

21. Steinman MA, Gonzales R, Linder JA, Landefeld CS. Changing use of antibiotics in community-based outpatient practice, 1991-1999. Ann Intern Med 2003; 138(7): 525-533.

22. McCaig LF, Besser RE, Hughes JM. Trends in antimicrobial prescribing rates for children and adolescents. JAMA 2002; 287(23): 3096-3102.

23. Steinman MA, Landefeld CS, Gonzales R. Predictors of broadspectrum antibiotic prescribing for acute respiratory tract infections in adult primary care. JAMA 2003; 289(6): 719-725.

24. Sharland $M$, Kendall $H$, Yeates D, et al. Antibiotic prescribing in general practice and hospital admissions for peritonsillar abscess, mastoiditis, and rheumatic fever in children: time trend analysis. $B M$, 2005; 331(7512): 328-329.

25. Ashworth M, Latinovic R, Charlton J, et al. Why has antibiotic prescribing for respiratory illness declined in primary care? A longitudinal study using the General Practice Research Database. $J$ Public Health (Oxf) 2004; 26(3): 268-274.

26. Frischer M, Heatlie H, Norwood J, et al. Trends in antibiotic prescribing and associated indications in primary care from 1993 to 1997. J Public Health Med 2001; 23(1): 69-73.

27. Fleming DM, Ross AM, Cross KW, Kendall H. The reducing incidence of respiratory tract infection and its relation to antibiotic prescribing. Br J Gen Pract 2003; 53(495): 778-783.

28. Smith S, Smith GE, Heatlie H, et al. Reducing variation in antibacterial prescribing rates for 'cough/cold' and sore throat between 1993 and 2001: regional analyses using the general practice research database. Public Health 2006; 120(8): 752-759.

29. Epic Database Research Company Ltd. Cegedim Strategic Data http://www.epic-uk.org/ (accessed 3 Sep 2009).

30. Gelfand J, Margolis, DJ, Dattani H. The UK General Practice Research Database. In: Strom BL (ed). Pharmacoepidemiology. Chichester: John Wiley \& Sons, Ltd, 2005: 337-346.

31. Metlay JP KJ. Failure to validate pneumococcal pneumonia diagnoses in the General Practice Research Database [abstract]. Pharmacoepidemiol Drug Saf 2003; 12: S163.
32. Hollowell J. The General Practice Research Database: quality of morbidity data. Popul Trends 1997; 87: 36-40.

33. Jick H, Jick SS, Derby LE. Validation of information recorded on general practitioner based computerised data resource in the United Kingdom. BMJ 1991; 302(6779): 766-768.

34. Wood F, Simpson S, Butler CC. Socially responsible antibiotic choices in primary care: a qualitative study of GPs' decisions to prescribe broad-spectrum and fluroquinolone antibiotics. Fam Pract 2007; 24(5): 427-434

35. Hing E, Cherry DK, Woodwell DA. National Ambulatory Medical Care Survey: 2004 summary. Adv Data 2006; 374: 1-33.

36. Schneeweiss S, Seeger JD, Maclure M, et al. Performance of comorbidity scores to control for confounding in epidemiologic studies using claims data. Am J Epidemiol 2001; 154(9): 854-864.

37. Schneeweiss S, Wang PS, Avorn J, et al. Consistency of performance ranking of comorbidity adjustment scores in Canadian and U.S. utilization data. J Gen Intern Med 2004; 19(5 Part 1): 444-450.

38. Putnam KG, Buist DS, Fishman P, et al. Chronic disease score as a predictor of hospitalization. Epidemiology 2002; 13(3): 340-346.

39. Zou G. A modified poisson regression approach to prospective studies with binary data. Am J Epidemiol 2004; 159(7): 702-706.

40. Miglioretti DL, Heagerty PJ. Marginal modeling of nonnested multilevel data using standard software. Am J Epidemiol 2007; 165(4): 453-463.

41. Hanley JA, Negassa A, Edwardes MD, Forrester JE. Statistical analysis of correlated data using generalized estimating equations: an orientation. [see comment]. Am J Epidemiol 2003; 157(4): 364-375.

42. McCullah P, Nelder JA. Generalized linear models. 2nd edn. London: Chapman and Hall, 1989.

43. Millar EV, O'Brien KL, Watt JP, et al. Effect of community-wide conjugate pneumococcal vaccine use in infancy on nasopharyngeal carriage through 3 years of age: a cross-sectional study in a high-risk population. Clin Infect Dis 2006; 43(1): 8-15.

44. O’Brien KL, Millar EV, Zell ER, et al. Effect of pneumococcal conjugate vaccine on nasopharyngeal colonization among immunized and unimmunized children in a community-randomized trial. J Infect Dis 2007; 196(8): 1211-1220.

45. Lipsitch M, O’Neill K, Cordy D, et al. Strain characteristics of Streptococcus pneumoniae carriage and invasive disease isolates during a cluster-randomized clinical trial of the 7-valent pneumococcal conjugate vaccine. J Infect Dis 2007; 196(8): 1221-1227.

46. Kyaw M, Lynfield R, Schafner W, et al. Effect of introduction of th pneumocococcal conjuate vaccine on drug-resistant Streptococcus pneumoniae. N Engl J Med 2006; 354(14): 1455-1463.

47. Finkelstein JA, Stille C, Nordin J, et al. Reduction in antibiotic use among US children, 1996-2000. Pediatrics 2003; 112(3 Part 1): 620-627.

48. Fleming DM, Cross KW, Barley MA. Recent changes in the prevalence of diseases presenting for health care. Br J Gen Pract 2005 55(517): 589-595.

49. Hing E, Cherry DK, Woodwell DA. National Ambulatory Medical Care Survey: 2004 Summary. Advance Data from Vital and Health Statistics 2006; 374. http://www.cdc.gov/nchs/data/ad/ad374.pdf (accessed 23 Mar 2009). 
Appendix 1. Acute upper respiratory tract infection diagnostic codes.

THIN Read code description

- Other acute upper respiratory infections

- Acute upper respiratory tract infection

- Upper respiratory infection NOS

- Upper respiratory tract infection NOS

- Acute nasopharyngitis

- Acute pharyngitis

- Throat infection - pharyngitis

- Acute pharyngitis NOS

- Sore throat NOS

- Acute bronchitis

- Bronchitis unspecified

NOS = not otherwise specified. 